\title{
Editorial: Im besten Sinne bodenständig
}

\author{
Andreas Klee · Gregor Prinzensing • Wladimir Sgibnev
}

Online publiziert: 26. November 2014

(C) Springer-Verlag Berlin Heidelberg 2014

Während das vorige Heft (2014-2015) sich globalen Ambitionen hingab und einen Bogen von Hockeroda bis nach New Orleans spannte, bleibt dieses Heft bodenständig aber nur, was den geographischen Rahmen anbelangt. Die Inhalte bieten, in gewohnter Manier, eine gute Mischung aus globaler Theorie, lokaler Kompetenz und einer großzügigen Prise Anwendungsbezug.

Eröffnet wird das Heft mit einem Diskussionsbeitrag von Dietrich Fürst zu ,Koordination und Führung in der Regionalplanung“. Der Autor will eine Debatte zur aktuellen und zukünftigen fachlichen und gesellschaftlichen Rolle von Planerinnen und Planern anregen - eine Stoßrichtung, die die Zeitschrift bereits im Augustheft mit dem Beitrag von Ingrid Krau mit ihrem Artikel zum Wandel des Berufsbilds der Stadt- und Raumplaner angestoßen hat. Fürst setzt sich zum Ziel, die Aufgaben der Regionalplanung im Diskussionsfeld der „Regional Governance“ zu verankern. Darüber hinaus legt der Autor den beteiligten Akteuren zahlreiche Handlungsempfehlungen ans Herz, insbesondere auf dem Gebiet der Kommunikation, die er als Schlüsselkompetenz planerischer Tätigkeit sieht.

Der darauffolgende Beitrag von Jörn Harfst und Peter Wirth befasst sich vor dem Hintergrund der Territorialen Agenda 2020 mit der „Bedeutung endogener Potenziale in klein- und mittelstädtisch geprägten Regionen“. Die Argu-

G. Prinzensing $(\bowtie)$

Erkner, Deutschland

E-Mail: prinzensing@arl-net.de

A. Klee

Hannover, Deutschland

W. Sgibnev

Leipzig, Deutschland mente der Autoren basieren auf einer Untersuchung von zwei Fallbeispielen - Mansfeld-Südharz (Sachsen-Anhalt) und Steirische Eisenstraße (Österreich). Die Identifizierung und Inwertsetzung endogener Potenziale kann trotz einer schwierigen sozioökonomischen Lage, so die Autoren, $\mathrm{zu}$ einer Entwicklung der Regionen beitragen. So würden endogene Potenziale z. B. regionale Entwicklungsprozesse anstoßen, Alleinstellungsmerkmale betonen und das Selbstwertgefühl der Regionen und ihrer Akteure stärken. Wir hoffen, dass es gelingt!

Das endogene Potenzial alternativer kultureller Einrichtungen steht im Mittelpunkt des Artikels von Christoph Mager, der damit einen „Beitrag zur Kritik ökonomischer Perspektiven auf Kreativräume“ leisten will. Der Autor kritisiert die vorherrschende Herangehensweise an kulturelle Infrastrukturen, die hierarchisierend wirkt und allein auf die ökonomische Verwertbarkeit kultureller Praktiken abzielt. Damit werden räumlich-gesellschaftliche Ungleichheiten produziert bzw. verstärkt und gleichzeitig Ankerpunkte des zivilgesellschaftlichen Engagements - vor allem in peripheren Räumen - untergraben. Damit ist dieser Beitrag auch als ein Plädoyer für einen Paradigmenwechsel bei der Betrachtung alternativer kultureller Einrichtungen zu verstehen.

Der Umgang mit Peripheralität ist auch ein wichtiges Element im Beitrag von Tine Köhler zu „Geoinformation in der kommunalen Abfallentsorgungs- und strategischen räumlichen Planung“. Angesichts steigender Infrastrukturkosten in durch Schrumpfung ,perforierten“ Räumen entwickelt die Autorin eine GIS-basierte Methode zur Senkung von Müllsammlungskosten durch die Einführung zentraler Sammelinseln. Darüber hinaus zeigt sie auf, inwiefern sich Abfallentsorgung als Steuerungsinstrument der Siedlungsentwicklung anbietet. Die Methode kann und soll dabei auch in Kommunen und Zweckverbänden außerhalb des südhessischen Fallbeispiels Verwendung finden. 
Der letzte wissenschaftliche Beitrag des Hefts greift das Großthema demographischer Wandel nicht unter dem Gesichtspunkt der Schrumpfung, sondern im Hinblick auf eine zunehmende Internationalisierung der Gesellschaft auf. Janina Welsch, Kerstin Conrad, Dirk Wittowsky und Ulrike Reutter wenden sich dem „Einfluss des Migrationshintergrundes auf die Alltagsmobilität im urbanen Raum“ zu. Die Autoren präsentieren eine Pilotstudie, die sich zum ersten Mal systematisch mit dem Mobilitätsverhalten von Menschen mit Migrationshintergrund befasst. Dabei beleuchten und diskutieren sie die erhobenen Daten zum Führerscheinbesitz, zur Pkw- und Fahrradausstattung sowie zur Nutzung der verschiedenen Verkehrsmittel.
Und zum Schluss eine Nachricht zum Wandel in eigener Sache: Andreas Klee vertritt im aktuellen Wintersemester die Professur für Raum- und Umweltplanung an der Martin-Luther-Universität Halle-Wittenberg. Für ein halbes Jahr zieht er sich daher von seiner operativen Tätigkeit als Schriftleiter von Raumforschung und Raumordnung zurück, steht aber weiterhin als Ansprechpartner für die Zeitschrift zur Verfügung. Vertreten wird er durch Gregor Prinzensing vom Leibniz-Institut für Regionalentwicklung und Strukturplanung (IRS). Den Leserinnen und Lesern sowie auch manchen Autorinnen und Autoren ist er sicher kein Unbekannter: 2010 und 2011 hatte er bereits die Schriftleitung der Zeitschrift inne. In diesem Sinne: Willkommen zurück! 